\title{
ON THE LITTLE-KNOWN HYPORHEIC BIODIVERSITY OF INDIA, WITH ANNOTATED CHECKLIST OF COPEPODS AND BATHYNELLACEANS (CRUSTACEA) AND A NOTE ON THE DISASTROUS IMPLICATIONS OF INDISCRIMINATE SAND MINING
}

Department of Zoology, Acharya Nagarjuna University, Nagarjunanagar, Andhra Pradesh 522510, India yrangareddyanu@gmail.com

\begin{abstract}
The vast and ecologically diversified hyporheic realm and the adjacent riparian areas of India have received scant attention from the standpoint of biodiversity studies. Analysis of about 2500 samples collected from the alluvial sediments of certain rivers and streams, besides some bores in the riparian zone, mainly in the coastal deltaic belt of the rivers Krishna and Godavari in Andhra Pradesh State during 2000-2012 yielded 41 copepod and bathynellacean species. Of these, 31 new species have been formally described during the ongoing studies whereas the remainder are previously known ones. An annotated checklist of all these taxa is presented, giving the type locality and other localities of occurrence, methods of sampling, chief references, and also some taxonomic and/or ecological remarks wherever necessary. The harpacticoid copepod family Parastenocarididae and the eumalacostracan order Bathynellacea are two significant, major groups of stygofauna that have been recorded for the first time from India. Both these groups and also some cyclopoid copepods have clear-cut Gondwanan lineages, representing the remnants of unique ancient fauna that require urgent attention from conservationists in order that the overall evolutionary history of the Indian biota is preserved. A note is also added on the devastating influence of the ongoing rampant sand mining activity on the hyporheic biodiversity.
\end{abstract}

Keywords: Bathynellacea, checklist, Copepoda, hyporheic ecosystem, riparian bores, sand mining impacts.

DOI: http://dx.doi.org/10.11609/JoTT.03734.5315-26 | ZooBank: urn:Isid:zoobank.org:pub:B72AB249-8CAA-4848-8E8F-A3CAC94EEB07

Editor: Paulo H.C. Corgosinho, Bairro Universitário, Frutal-MG, Brazil.

Date of publication: 26 January 2014 (online \& print)

Manuscript details: Ms \# 03734 | Received 03 August 2013 | Final received 07 December 2013 | Finally accepted 31 December 2013

Citation: Ranga Reddy, Y. (2014). On the little-known hyporheic biodiversity of India, with annotated checklist of copepods and bathynellaceans (Crustacea) and a note on the disastrous implications of indiscriminate sand mining. Journal of Threatened Taxa 6(1):5315-5326; http://dx.doi.org/10.11609/JoTT.o3734.5315-26

Copyright: ( ) Ranga Reddy 2014. Creative Commons Attribution 3.0 Unported License. JoTT allows unrestricted use of this article in any medium, reproduction and distribution by providing adequate credit to the authors and the source of publication.

Funding: Department of Science \& Technology, Ministry of Science \& Technology, Government of India, New Delhi, under a Major Research Project (SR/SO/AS-21/2011)

Competing Interest: The authors declare no competing interests.

Author Details: The author is an Emeritus Fellow and specializes in the taxonomy and biogeography of freshwater free-living copepods and also groundwater copepods and bathynellaceans (Crustacea) of India.

Acknowledgements: The author is grateful to the authorities of Acharya Nagarjuna University for extending necessary facilities. The author also expresses his grateful thanks to the anonymous reviewers for providing several critical comments. 


\section{INTRODUCTION}

To save the earth's fast-depleting biodiversity, which is due primarily to habitat loss and impairment, has become a matter of increasing concern for scientists and governments all over the world. What is even more disconcerting today is that certain unique and asyet unexplored habitats are imperiled even before the study of their biodiversity and functions has begun in right earnest. One such greatly threatened habitat is the hyporheic habitat present within the sandy banks of rivers and streams. In India, scant attention, if any, has been paid to the study of hyporheic ecosystems. This paper gives, after briefly referring to the general biodiversity and functions of the hyporheic zone, an annotated checklist of the hyporheic copepod and bathynellacean crustaceans (size c. $1 \mathrm{~mm}$ ). This checklist is based on the taxonomic and biogeographic studies carried out during the last decade or so, mostly in the coastal deltaic belt of the rivers Krishna and Godavari in Andhra Pradesh State. Stringent legislative measures are suggested for protecting these precious sanddwellers and their home, now under the devastating impact of excessive sand extraction. Incidentally, the stray hypogean copepod and bathynellacean species encountered in the riparian bores and two caves are also included in the checklist.

The term hyporheic, derived from Greek rootshypo, meaning under or beneath, and rheos, meaning a stream, was first used by the Romanian hydrologist Orghidan (1959). It originally refers to the alluvial sediments that extend vertically and laterally from the river channel, giving rise to 'a rich and unique ecosystem'. Demonstrating the double influence of groundwater and stream properties on this ecosystem, Orghidan distinguished it from other groundwater habitats. And the subsequent pioneering work by Stanford \& Ward (1993) proposes to integrate the stream channel and hyporheic systems into a river continuum conceptthe hyporheic corridor. Essentially, the hyporheic zone constitutes 'a spatially and temporally dynamic ecotone' (Feris et al. 2003), sandwiched between the surface water and groundwater ecosystems. This 'critical interface' extends from the substrate surface to a depth of about $50 \mathrm{~cm}$, below which lies the phreatic or groundwater regime (Pennak 1940). Its functional role is governed by such properties as its elasticity, permeability, biodiversity, and connectivity (Gibert et al. 1990; Vervier et al. 1992) in close interaction with the geomorphology, geohydrology, landscape use and the buffering ecosystems along the river corridor. It is in this zone that hydrological, ecological and biogeochemical processes interact, influencing key ecosystem processes such as primary productivity and nutrient cycling (Mulholland \& Webster 2010). Hence, the findings concerning the functional significance of the hyporheic zone are of crucial importance in floodplain management and restoration (Boulton et al. 2010). Overall, the hyporheic science, which is a vital facet of groundwater ecology and 'a topic of great practical relevance' to regulators and policy makers, has been recognized in the West as a fascinating, multidisciplinary field that combines methods, concepts and data from hydrogeology, geochemistry, microbiology and aquatic ecology (Larned 2012). Besides being the home of rich biodiversity, the hyporheic zone endows us with a number of 'ecological goods and services' such as the following: offers a spawning ground and refuge for certain fishes (salmon, etc.) and rooting zone for aquatic plants; controls the flux and location of water exchange between streams and subsurface; acts as a buffer zone for the attenuation of certain pollutants by biodegradation, sorption and mixing; provides an important zone for biogeochemical cycling of carbon, energy and nutrients; forms a functional sink/source for fine organic detritus and other sediments; and moderates water temperature against heat and freezing (Environment Agency 2009).

As to the biodiversity, the hyporheic zone supports a heterotrophic assemblage of both interstitial and benthic community (hyporheos) of diverse groups of organisms including some ancient and rare relictual Gondwanan lineages that are now absent from the surface waters. The hyporheic biodiversity is generally composed of Protista (Ciliophora, Sarcomastigophora), Gastrotricha, Tardigrada, Oligochaeta (Aelosomatidae), Annelida (Potamodrilidae), Insecta (Collembola, Ephemeroptera, Odonata, Plecoptera, Trichoptera, Coleoptera, Diptera), Rotifera, Acari, Crustacea (Copepoda, Ostracoda, Amphipoda, Isopoda, Cladocera, Syncarida, Thermosbaenacea), Mollusca (Aplacophora, Bivalvia, Gastropoda), Platyhelminthes (Turbellaria) and Nematoda (Hakenkamp \& Palmer 1999). The obligate interstitial animals (stygobites) are small, elongated and vermiform, blind, unpigmented, and have reduced limbs and/or elongated sonsorial structures, which compensate for the lack of vision, and produce but a few eggs and develop slowly (Danielopol et al. 1994). Based on their body size, the hyporheic organisms are often grouped into three categories: micro- $(<50 \mu \mathrm{m})$, meio$(50-1000 \mu \mathrm{m})$, and macro- $(>1000 \mu \mathrm{m})$ elements. Given the well-known 'ecotone edge effects' (Odum 1971), the hyporheic systems can support extremely large densities 
of meiofauna (although low densities and high diversity is the general rule), which eventually may equal or even exceed those in marine systems (Palmer 1990; Borchardt \& Bott 1995), and provide a vital trophic link between bacteria, meiofauna and macrofauna. The meiofauna feed on benthic bacteria and algae, influencing the microbial and algal community, as well as biofilm and particulate organic matter dynamics (Hakenkamp et al. 2002). Their selective feeding on bacteria and algae could as well influence stoichiometry (e.g., $\mathrm{C}, \mathrm{N}$, and $\mathrm{P}$ ratios) of stream sediments (Elser et al. 1996; Hessen 1997). The fauna, in turn, constitute an important part of the diet of many freshwater fishes (Williams 1981; McNicol et al. 1985; Brown et al. 1989; Schmid-Araya \& Schmid 2000; Ranga Reddy 2001) and predaceous invertebrates (Benke \& Wallace 1980; Schmith \& Smock 1992; SchmidAraya \& Schmid 2000). The usefulness of hyporheic fauna as indicators (biosensors) of anthropogenic impacts such as pollution has also been documented for certain groups (Notenboom et al. 1994; Boulton $2000 a, b)$. Furthermore, the very ancient crustaceans such as bathynellaceans have long been recognized as suitable objects for understanding the history of the earth's crust and biological speciation (Schminke 1974; Schram 1977, 2008) (see Discussion).

\section{MATERIALS AND METHODS}

About 2500 core samples collected mainly from the alluvial sediments mostly in the coastal deltaic belt of the rivers Krishna and Godavari and also from the riparian/ phreatic bores in Andhra Pradesh State (exceptions: only a single sample each from the river Sutlej in Himachal Pradesh State and River Muvattupuzha in Kerala State, and two samples from cave pools) during 2000-2012 by adopting the following methods:

(1) Karaman \& Chappuis method (Chappuis 1942): This method, the most frequently used one, entails digging a few holes of varying depths $(10-30 \mathrm{~cm})$ a few meters apart from one another in the alluvial deposits next to a stream or river, and sampling the subsurface water seeping into the pits. Each time the sample was filtered through bolting-silk plankton net (mesh size $70 \mu \mathrm{m})$, and the filtrate fixed in $5 \%$ formaldehyde in plastic vials.

(2) Coring and filtration method: A rigid PVC tube (length c. $70 \mathrm{~cm}$, diameter c. $10 \mathrm{~cm}$ ) was used to extract cores from the sediment surface to a depth of $10-50 \mathrm{~cm}$ from both exposed and submerged parts of stream/river banks. At each site, the core samples were pooled in a bucket, filled with the water from the sampling spot and stirred vigorously. The supernatant was filtered and fixed as mentioned above.

(3) Direct filtration of water from farm bores in the riparian zone: Specimens were collected by filtering the water when it was pumped out of farm bores (depth c. $10 \mathrm{~m}$ ) adjacent to rivers. Filtering was done manually by holding a bolting-silk plankton net (mesh size $70 \mu \mathrm{m}$ ) against the water current for 20-30 minutes at each time of sampling. The filtrate was fixed as before.

In the laboratory, the specimens studied were sorted into $70 \%$ alcohol and later transferred into glycerol; their morphology was studied using the general methods of microscopy in vogue. For the sake of completeness of the checklist of the species that are essentially hypogean, three cavernicolous species, collected by Coring and filtration method, are also included. Abbreviations used: $A P=$ Andhra Pradesh State; EGD = East Godavari District; R. = River.

\section{RESULTS}

Phylum Arthropoda

Subphylum Crustacea Brünich, 1772

Class Maxillopoda Dahl, 1956

Subclass Copepoda Milne-Edwards, 1840

Order Cyclopoida Burmeister, 1834

Subfamily Cyclopinidae Dana, 1846

Genus Allocyclopina Kiefer, 1954

1. Allocyclopina inopinata Defaye \& Ranga Reddy, 2008

Type locality: R. Godavari at Kotipalli Village $\left(18^{\circ} 85^{\prime} \mathrm{N}\right.$ \& $\left.82^{\circ} 02^{\prime} \mathrm{E} ; 10 \mathrm{~m}\right)$ near Ramachandrapuram Town, EGD., AP.

Other localities: None.

Sampling methods: Karaman \& Chappuis method, and direct filtration method.

Reference: Defaye \& Ranga Reddy 2008: 1119-1141.

Remark: Tolerates brackish conditions.

Family Cyclopidae Rafinesque, 1815

Subfamily Eucyclopinae Kiefer, 1927

Genus Paracyclops Claus, 1893

\section{Paracyclops fimbratus (Fischer, 1853)}

Type locality: P. Dudegofka, St. Petersburg $\left(59^{\circ} 53^{\prime} 39^{\prime \prime} \mathrm{N} \& 30^{\circ} 15^{\prime} 51^{\prime \prime} \mathrm{E} ; 5 \mathrm{~m}\right)$, Russia.

Other localities: Europe and Asia extending eastwards to include Turkey, Palestine, China, Japan and India and widely distributed throughout the Palaearctic region (Karaytug 1999). Found but rarely in the interstitial 
samples of R. Krishna at Vijayawada city $\left(16^{0} 31^{\prime} 8.50^{\prime \prime} \mathrm{N}\right.$ \& 80037'17.38"E; 88m), AP.

Sampling method: Coring and filtration method.

References: Karaytug \& Boxshall 1998: 563-602; Karaytug 1999: 30-43.

Remark: Morphologically highly variable species; generally epibenthic.

Subfamily Cyclopinae Kiefer, 1927

Genus Haplocyclops Kiefer, 1952

Subgenus Kiefercyclops Karanovic \& Ranga Reddy, 2005

3. Haplocyclops (Kiefercyclops) fiersi Karanovic \& Ranga Reddy, 2005.

Type locality: Bore well on Acharya Nagarjuna University campus ( $16^{\circ} 22^{\prime} 41^{\prime \prime} \mathrm{N} \& 80^{\circ} 31^{\prime} 39.4^{\prime \prime} \mathrm{E}$; $\left.19.8 \mathrm{~m}\right)$, $13 \mathrm{~km}$ from Guntur Town, in the riparian zone of Nambur canal of R. Krishna, AP.

Other localities: None.

Sampling method: Direct filtration method.

Reference: Karanovic \& Ranga Reddy 2005: 83-92.

Remark: Most reduced free-living cyclopoid; generally confined to riparian bore wells.

\section{Genus Rybocyclops, 1982}

\section{Rybocyclops dussarti Ranga Reddy \& Defaye,} 2008

Type locality: Agriculture bore, in the riparian zone of Gundlakamma R., at Chollaveedu village $\left(15^{\circ} 31^{\prime} 39^{\prime \prime} \mathrm{N}\right.$ $\left.78^{0} 56^{\prime} 56^{\prime \prime} \mathrm{E} ; 231 \mathrm{~m}\right)$, Racharla Mandal of Prakasam District, AP.

Other locality: Bore well, in the riparian/phreatic zone of Gundlakamma R., at Araveetikota Village $\left(15^{\circ} 34^{\prime} 49^{\prime \prime} \mathrm{N} 78^{\circ} 55^{\prime} 56^{\prime \prime} \mathrm{E}\right.$; $\left.235 \mathrm{~m}\right)$ of Racharla Mandal of Prakasam District, AP.

Sampling method: Direct filtration method.

Reference: Ranga Reddy \& Defaye 2008: 41-49.

Remark: Has clear-cut Gondwanan affinities with the Madagascan congener, Rybocyclops pauliani (Lindberg 1954) (see Ranga Reddy 2011b).

Order Harpacticoida Sars, 1903

Family Phyllognathopodidae Gurney, 1932

Genus Phyllognathopus Mrazek, 1893

\section{Phyllognathopus viguieri (Maupas, 1892)}

Type locality: Not known.

Other localities: Cosmopolitan species; present record from the hyporheic habitat of R. Godavari at Rajahmundry $\left(16^{\circ} 59^{\prime} \mathrm{N} \& 81^{\circ} 47^{\prime} \mathrm{E} ; 14 \mathrm{~m}\right)$, EGD, AP.

Sampling method: Coring and filtration method.

References: Karanovic \& Ranga Reddy 2004a: 122131; Galassi et al. 2011: 4-17.
Remark: Morphologically highly variable species; possibly a cryptic morphospecies (Wells 2011).

Genus Neophyllognathopus Galassi \& De Laurentiis, 2011

6. Neophyllognathopus bassoti (Rouch, 1972)

Type locality: Interstitial of Lake Wisdom (50 $10^{\prime} 55^{\prime \prime} \mathrm{S}$ \& $147^{0} 7^{\prime} 6^{\prime \prime} \mathrm{E} ; 400 \mathrm{~m}$ ) on Long Island, Papua New Guinea.

Other localities: Wells on Bantayan Island $\left(11^{0} 13^{\prime} 15^{\prime \prime} \mathrm{N} \& 123^{\circ} 44^{\prime} 45^{\prime \prime} \mathrm{E}\right.$; $\left.30 \mathrm{~m}\right)$ of the Philippines; present records from bore wells in Brindavan Gardens $\left(16^{\circ} 18^{\prime} \mathrm{N} \& 80^{\circ} 29^{\prime} \mathrm{E}\right.$; 33m) of Guntur Town in Guntur District, and Kandukur Town (150 $\left.15^{\prime} \mathrm{N} \& 7^{\circ} 47^{\prime} \mathrm{E} ; 632 \mathrm{~m}\right)$ in Prakasam District, AP.

Sampling method: Direct filtration method.

References: Rouch 1972: 148-155; Bruno \& Cottarelli, 1999: 521-528; Karanovic \& Ranga Reddy 2004b: 247259; Galassi et al. 2011: 32-43.

Family Ameiridae Boeck, 1865

Subfamily Ameirinae Boeck, 1865

Genus Nitokra Boeck, 1865

7. Nitokra ?lacustris (Schmankevitch, 1895)

Type locality: Not available.

Other localities: Widely distributed in the world (see Dussart \& Defaye 1990); present record from R. Krishna at Vijayawada City $\left(16^{0} 31^{\prime} 8.50^{\prime \prime} \mathrm{N}\right.$ \& 80 $37^{\prime} 17.38^{\prime \prime} \mathrm{E}$; $11.88 \mathrm{~m}), \mathrm{AP}$

Reference: Lang 1948: 812-14.

Sampling method: Coring and filtration method.

Remark: The morphology shows certain characters that are suggestive of a new subspecies rather than the typical form (Ranga Reddy unpubl.). Rare in the interstitial.

Family Canthocamptidae Brady, 1880

Subfamily Canthocamptiinae Brady, 1880

Genus Elaphoidella Chappuis, 1928

\section{Elaphoidella crassa Chappuis, 1954}

Type locality: Mawsmai Cave near Cherrapunji $\left(25^{\circ} 18^{\prime} \mathrm{N} \& 91^{\circ} 42^{\prime} \mathrm{E} / 25.30^{\circ} \mathrm{N} \& 91.70^{\circ} \mathrm{E}\right.$; $1,484 \mathrm{~m})$, Meghalaya State.

Other localities: None.

Sampling method: Not known.

Reference: Chappuis 1954: 218-220.

Remark. First cavernicolous copepod species from India.

Genus Mesochra Boeck, 1865

9. Mesochra wolskii Jakubisiak, 1933

Type locality: Lagoon Matanzas $\left(23^{\circ} 03^{\prime} 4^{\prime \prime} \mathrm{N}\right.$ \& 
81034'31"W; 20m), Cuba.

Other localities: Different localities in North and South Atlantic, Pacific, and Indian Oceans. Present record from R. Krishna at Vijayawada City $\left(16^{0} 31^{\prime} 8.50^{\prime \prime} \mathrm{N}\right.$ \& 80037'17.38" E; 11.88m), AP.

Sampling method: Coring and filtration method.

Reference: Fiers \& Rutledge 1990: 108-111.

Remark: A predominantly marine taxon; well established in R. Krishna at Vijayawada City; rare in the interstitial.

\section{Genus Cletocamptus Schmankevitsch, 1875}

\section{Cletocamptus deitersi (Richard, 1887)}

Type locality: Naposta Grande R., Argentina.

Other localities: R. Krishna at Vijayawada City

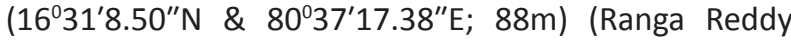
2001); Lake Kolleru $\left(16^{0} 39^{\prime} \mathrm{N} \& 81^{\circ} 13^{\prime} \mathrm{E} ; 3.26 \mathrm{~m}\right)$ (Ranga Reddy \& Radhakrishna 1979), AP; widely distributed in the world (Dussart \& Defaye 1990).

Sampling method: Coring and filtration method.

References: Lang 1948: 1278-1280; Hamond 1973: 414-417.

Remarks: Currently considered as a species inquirenda (Gómez et al. 2004); a highly euryhaline species.

Family Diosaccidae Sars, 1906

Genus Neomiscegenus Karanovic \& Ranga Reddy, 2004

11. Neomiscegenus indicus Karanovic \& Ranga

\section{Reddy, 2004}

Type locality: Groundwater runoff on the southern bank of R. Krishna at Vijayawada City $\left(16^{0} 31^{\prime} 8.50^{\prime \prime} \mathrm{N}\right.$, $80^{\circ} 37^{\prime} 17.38^{\prime \prime} \mathrm{E} ; 11.88 \mathrm{~m}$ ) in AP.

Other localities: None.

Sampling method: Direct filtration method.

Reference. Karanovic \& Ranga Reddy 2004b: 246260; Wells 2007: 531.

Remark: Seems to prefer the aquifers in the riparian zone.

Family Ectinosomatidae Sars, 1903

Genus Rangabradya Karanovic \& Pesce, 2001

\section{Rangabradya indica Karanovic \& Pesce, 2001}

Type locality: Freshwater bore well in Brindavan Gardens $\left(16^{\circ} 18^{\prime} \mathrm{N} \& 80^{\circ} 29^{\prime} \mathrm{E} ; 33 \mathrm{~m}\right)$ of Guntur Town in Guntur District, AP.

Other localities: None.

Sampling method: Direct filtration method.

Reference: Karanovic \& Pesce 2001: 282-290; Wells 2011: 382, 388
Family Miraciidae Dana, 1846

Subfamily Steheliinae Brady, 1880

Genus Delavalia Brady, 1880

\section{Delavalia madrasensis (Wells, 1971)}

Type locality: Estuarine beaches of the Vellar R. near Porto Novo $\left(6^{0} 29^{\prime} 47^{\prime \prime} \mathrm{N} \& 2^{0} 36^{\prime} 12^{\prime \prime} \mathrm{E} ; 40 \mathrm{~m}\right)$, Tamilnadu (erstwhile Madras State).

Other localities: Rambha Bay, Chilka Lake $\left(19^{\circ} 41^{\prime} 39^{\prime \prime} \mathrm{N} \& 85^{\circ} 18^{\prime} 24^{\prime \prime} \mathrm{E} / 19.69417^{\circ} \mathrm{N}\right.$ \& $85.30667^{\circ} \mathrm{E}$; $8 \mathrm{~m})$, Odisha State, and intertidal sand on Long Island, Middle Andaman Islands (Wells \& Rao 1987); present record from R. Krishna at Vijayawada $\left(16^{0} 31^{\prime} 8.50^{\prime \prime} \mathrm{N}\right.$ \& 80 37'17.38'"E; 11.88m), AP.

Sampling method: Coring and sampling method.

References: Wells 1971: 509-510 (female only as Stenhelia madrasensis); Radhakrishna \& Ranga Reddy 1978: 152-158 (both sexes under the synonym Stenhelia krishnensis); Wells 2007: 548.

Remarks.: A highly euryhaline species, well established in R. Krishna.

Family Laophontidae T. Scott, 1905

Subfamily Laophontinae T. Scott, 1905

Genus Folioquinpes Fiers \& Rutledge, 1990

\section{Folioquinpes chathamensis (Sars, 1905)}

Type locality: Chatham Islands $\left(44^{\circ} 02^{\prime} \mathrm{S} \& 176^{\circ} 26^{\prime} \mathrm{W}\right.$ / $44.033^{\circ} \mathrm{S} \& 176.433^{\circ} \mathrm{W} ; 294 \mathrm{~m}$ ), an archipelago in the Pacific Ocean.

Other localities: Cape Town $\left(33^{\circ} 55^{\prime} 31^{\prime \prime} \mathrm{S} \& 18^{\circ} 25^{\prime} 26^{\prime \prime} \mathrm{E}\right.$; $1,590.4 \mathrm{~m})$, Africa; Chilka Lake $\left(19^{\circ} 41^{\prime} 39^{\prime \prime} \mathrm{N} \& 8^{\circ} 18^{\prime} 24^{\prime \prime} \mathrm{E}\right.$ ( $19.69417^{\circ} \mathrm{N}$ \& $85.30667^{\circ} \mathrm{E} ; 8 \mathrm{~m}$ ), India; New Zealand (Dussart \& Defaye 1990); present record from R. Krishna at Vijayawada City $\left(16^{0} 31^{\prime} 8.50^{\prime \prime} \mathrm{N} \& 8^{\circ} 37^{\prime} 17.38^{\prime \prime} \mathrm{E}\right.$; $11.88 \mathrm{~m})$.

Sampling method: Coring and filtration method.

References: Lang 1948: 1119; Sewell 1924: 830-832, pl. 57, fig. 2; Wells 2011: 431, 440, 460.

Remarks: Mostly a brackish form, but occurring in the purely freshwater condition of R. Krishna.

Family Parastenocarididae Chappuis, 1940

Genus Parastenocaris Chappuis, 1940

\section{Parastenocaris curvispinus Enckell, 1970}

Type locality: “W. Prov., Kalutara [6 $34^{\prime} 59^{\prime} \mathrm{N}$ \& $\left.79^{\circ} 57^{\prime} 33^{\prime \prime} \mathrm{E} ; 3 \mathrm{~m}\right], 25$ miles, S. Colombo. Long sandbank in the estuary of the river".

Other localities. R. Krishna at Vijayawada $\left(16^{0} 31^{\prime} 8.50^{\prime \prime} \mathrm{N} \& 80^{\circ} 37^{\prime} 17.38^{\prime \prime} \mathrm{E} ; 11.88 \mathrm{~m}\right)$, R. Godavari at Rajahmundry $\left(16^{\circ} 59^{\prime} \mathrm{N} \& 81^{\circ} 47^{\prime} \mathrm{E} ; 14 \mathrm{~m}\right)$, and R. Pennar at Chennur Village $\left(14.14733^{\circ} \mathrm{N} \& 79.847712^{\circ} \mathrm{E}\right.$; $115 \mathrm{~m})$ near Kadapa Town in Andhra Pradesh State; R. 


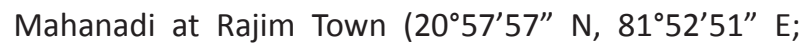
$281 \mathrm{~m})$, R. Yamuna at Champaran, and R. Indravati at Chitrakoot Waterfalls in Chhattisgarh State; R. Periyar at Kalady Village $\left(10^{\circ} 10^{\prime} \mathrm{N} \& 76^{\circ} 26^{\prime} \mathrm{E}\right), \mathrm{R}$. Bharatpuzha at Cheruthuruthi $\left(10^{\circ} 44^{\prime} \mathrm{N} \& 76^{\circ} 17^{\prime} \mathrm{E}\right)$, and R. Muvattupuzha at Muvattupuzha $\left(9^{\circ} 96^{\prime} 70^{\prime \prime} \mathrm{N} \& 76^{\circ} 58^{\prime} 30^{\prime \prime} \mathrm{E} ; 28 \mathrm{~m}\right)$ in Kerala State.

Sampling methods: Karaman \& Chappuis method, and Coring and filtration method.

References: Enckell 1970: 553; Ranga Reddy \& Defaye 2007: 9-17; Cottarelli et al. 2010: 488.

Remarks: A euryhaline species, most widespread and common in the hyporheic habitats of the peninsular India, the present range extending from south-western Sri Lanka to central India.

\section{Parastenocaris gayatri Ranga Reddy, 2001}

Type locality: Interstitial of R. Krishna at Vijayawada city $\left(16^{0} 31^{\prime} 8.50^{\prime \prime} \mathrm{N} \& 80^{\circ} 37^{\prime} 17.38^{\prime \prime} \mathrm{E} ; 11.88 \mathrm{~m}\right)$.

Other localities: River Krishna at Amaravati $\left(16^{\circ} 34^{\prime} 48^{\prime \prime} \mathrm{N} \& 80^{\circ} 21^{\prime} 36^{\prime \prime} \mathrm{E} ; 14 \mathrm{~m}\right)$ in Guntur District and R. Godavari at Rajahmundry, EGD, AP.

Sampling methods: Karaman \& Chappuis method and Coring and filtration method.

Reference: Ranga Reddy 2001: 708-716; Karanovic 2005: 370; Cottarelli et al. 2010: 487; Schminke 2010: 350.

Remarks: A fairly common species in the R. Krishna and R. Godavari.

\section{Parastenocaris savita Ranga Reddy, 2001}

Type locality: Interstitial of R. Krishna at Vijayawada City $\left(16^{0} 31^{\prime} 8.50^{\prime \prime} \mathrm{N} \& 80^{\circ} 37^{\prime} 17.38^{\prime \prime} \mathrm{E} ; 11.88 \mathrm{~m}\right)$, A. P.

Other localities: R. Krishna at Amaravati $\left(16^{0} 34^{\prime} 48^{\prime \prime} \mathrm{N}\right.$ \& 80 $21^{\prime} 36^{\prime \prime} \mathrm{E} ; 14 \mathrm{~m}$ ) in Guntur District and R. Godavari at Rajahmundry $\left(16^{\circ} 59^{\prime} \mathrm{N} \& 81^{\circ} 47^{\prime} \mathrm{E} ; 14 \mathrm{~m}\right)$, EGD.

Sampling methods: Karaman \& Chappuis method, and Coring and filtration method.

References: Ranga Reddy 2001: 716-722; Ranga Reddy \& Defaye 2007: 22-24. Schminke 2010: 350.

\section{Parastenocaris mahanadi Ranga Reddy \&} Defaye, 2007

Type locality: R. Mahanadi at Rajim Town $\left(20^{\circ} 57^{\prime} 57^{\prime \prime} \mathrm{N}\right.$ \& $\left.81^{0} 52^{\prime} 51^{\prime \prime} \mathrm{E} ; 281 \mathrm{~m}\right)$, Chhattisgarh State.

Other locality: None.

Sampling method: Coring and filtration method.

References: Ranga Reddy \& Defaye 2007: 2-9, $22-$ 24; Schminke 2010: 350.

Remark: A rare species.

\section{Parastenocaris muvattupuzha Ranga Reddy \&} Defaye, 2009

Type locality: R. Muvattupuzha at Muvattupuzha Town ( $\left.9^{0} 58^{\prime} 01^{\prime \prime} \mathrm{N} \& 76^{0} 34^{\prime} 59^{\prime \prime} \mathrm{E} ; 15 \mathrm{~m}\right)$, Kerala State.

Other localities. None.

Sampling method: Coring and filtration method.

Reference: Ranga Reddy \& Defaye 2009: 32-43; Schminke 2010: 350; Cottarelli et al. 2010: 488.

\section{Parastenocaris kotumsarensis Ranga Reddy \&} Defaye, 2009

Type locality: Kotumsar cave located on the bank of the R. Kanger, flowing through the Kanger Valley National Park (18052'09"N \& 81056'05"E; 560m) near Jagdalpur Town, Chhattisgarh State.

Other localities: None.

Sampling methods: Coring and filtration method and Direct filtration method.

Reference: Ranga Reddy \& Defaye 2009: 43-51.

Remark: First cavernicolous parastenocaridid species from India.

\section{Parastenocaris sutlej Ranga Reddy, 2011}

Type locality: R. Sutlej at Tattapani $\left(31^{0} 14^{\prime} 56^{\prime \prime} \mathrm{N}\right.$ $\left.77^{\circ} 05^{\prime} 10^{\prime \prime} \mathrm{E} ; 656 \mathrm{~m}\right)$, Himachal Pradesh State.

Other localities: None.

Sampling method: Coring and filtration method.

Reference: Ranga Reddy 2011a: 464-473.

Remark: First parastenocaridid species from a Himalayan River.

22. Parastenocaris gundlakamma Ranga Reddy, 2011

Type locality: Gundlakamma River pond at Nemiligundla Sri Ranganayakaswamy Temple (15030.916'N \& 78052.155'E; 289m) near Giddalur Town, Prakasam District, AP.

Other localities: None.

Sampling method: Coring and filtration method.

Reference: Ranga Reddy 2011a: 474-482.

23. Parastenocaris tirupatiensis Ranga Reddy, 2011

Type locality: Bore well at S.V. University campus $\left(13^{\circ} 37^{\prime} 44^{\prime \prime} \mathrm{N} \& 7^{\circ} 23^{\prime} 58^{\prime \prime} \mathrm{E} ; 162 \mathrm{~m}\right)$, Tirupati Town, phreatic/ perhaps the riparian zone of Swarnamukhi R.

Other localities: None.

Sampling method: Direct filtration method.

Reference: Ranga Reddy 2011c: 21-29. 
Genus Kinnecaris Jakobi, 1972

24. Kinnecaris godavari Ranga Reddy \& Schminke, 2009

Type locality: R. Godavari at Rajahmundry $\left(16^{\circ} 59^{\prime} \mathrm{N}\right.$

\& $\left.81^{\circ} 47^{\prime} \mathrm{E} ; 14 \mathrm{~m}\right), \mathrm{EGD}, \mathrm{AP}$.

Other localities: None.

Sampling method: Coring and filtration method.

Reference: Ranga Reddy \& Schminke 2009a: 312325; Schminke 2010: 350; Cottarelli et al. 2010: 488.

Genus Siolicaris Jakobi, 1972

\section{Siolicaris sandhya (Ranga Reddy, 2001)}

Synonym: Parastenocaris sandhya Ranga Reddy, 2001.

Type locality: Interstitial of R. Krishna at Vijayawada City $\left(16^{0} 31^{\prime} 8.50^{\prime \prime} \mathrm{N} \& 80^{\circ} 37^{\prime} 17.38^{\prime \prime} \mathrm{E} ; 11.88 \mathrm{~m}\right)$, AP.

Other localities: R. Krishna at Amaravati $\left(16^{0} 34^{\prime} 48^{\prime \prime} \mathrm{N}\right.$

\& 80 21'36"E; 14m) in Guntur District, and R. Godavari at Rajahmundry $\left(16^{0} 59^{\prime} \mathrm{N} \& 81^{\circ} 47^{\prime} \mathrm{E} ; 14 \mathrm{~m}\right)$, EGD, AP.

Sampling methods: Karaman \& Chappuis method and Coring and filtration method.

References: Ranga Reddy 2001: 723-730; Ranga Reddy \& Defaye 2007: 22-24. Corgosinho et al. 2012: 59-65.

Remarks: This is a rare species and restricted to the

R. Krishna and R. Godavari, AP.

Class Malacostraca Latreille, 1802

Subclass Eumalacostraca, 1892

Order Bathynellacea Chappuis, 1915

Family Bathynellidae Grobben, 1905

Genus Serbanibathynella Ranga Reddy \& Schminke, 2005

26. Serbanibathynella primaindica Ranga Reddy \& Schminke, 2005

Type locality: Farm bore at Tadepalli Village $\left(16^{\circ} 41^{\prime} 32^{\prime \prime} \mathrm{N} \& 82^{\circ} 02^{\prime} 24^{\prime \prime} \mathrm{E} ; 12.5 \mathrm{~m}\right.$; water temperature $26^{\circ} \mathrm{C}$; $\mathrm{pH} 8.0$ ), $3 \mathrm{~km}$ from Vijayawada, in the riparian zone of R. Krishna, AP.

Other localities: Four other bores within a radius $2 \mathrm{~km}$ from the type locality.

Sampling method: Direct filtration method.

References: Ranga Reddy \& Schminke 2005b: 25-30;

Camacho 2006: 19.

Remark: Mostly confined to riparian bore wells.

Genus Indobathynella Ranga Reddy \& Totakura, 2012

27. Indobathynella prehensilis Ranga Reddy \& Totakura, 2012

Type locality: Farm bore at Ravulapalem Village (16 $06^{\prime} 33.4^{\prime \prime} \mathrm{N} \& 8^{\circ} 46^{\prime} 49.9^{\prime \prime} \mathrm{E}$; $37 \mathrm{~m}$; depth c. $10 \mathrm{~m}$; water temperature $27^{\circ} \mathrm{C} ; \mathrm{pH} 7.0$ ), c. $30 \mathrm{~km}$ from Rajahmundry, in the riparian zone of R. Godavari, EGD, AP.

Other localities: Farm bore, Chintalapudi Village $\left(16^{\circ} 02^{\prime} 23.8^{\prime \prime} \mathrm{N} \& 80^{\circ} 32^{\prime} 35.4^{\prime \prime} \mathrm{E} ; 36.5 \mathrm{~m}\right), \sim 5 \mathrm{~km}$ from Nidubrolu Town, Guntur District, farm bore, Peravaram Village $\left(16^{0} 53^{\prime} 42.0^{\prime \prime} \mathrm{N} \& 8^{\circ} 45^{\prime} 09.8^{\prime \prime} \mathrm{E}\right.$; $17.7 \mathrm{~m}$; water temperature $\left.26^{\circ} \mathrm{C} ; \mathrm{pH} 7.0\right), \sim 20 \mathrm{~km}$ from Rajahmundry, EGD, AP.

Sampling method: Direct filtration method.

Reference: Ranga Reddy \& Totakura 2012a: 281-293.

Family Parabathynellidae Noodt, 1965

Genus Atopobathynella Schminke, 1973

28. Atopobathynella operculata Ranga Reddy, Drewes \& Schminke, 2008

Type locality: R. Godavari at Rajahmundry Town $\left(16^{0} 59^{\prime} \mathrm{N} \& 81^{\circ} 47^{\prime} \mathrm{E} ; 14 \mathrm{~m}\right), \mathrm{EGD}, \mathrm{AP}$.

Other localities: None.

Sampling method: Coring and filtration method.

Reference: Ranga Reddy, Drewes \& Schminke 2008: 52-60.

Genus Chilibathynella Noodt, 1964

29. Chilibathynella kotumsarensis Ranga Reddy, 2006

Type locality: Kutumsar cave located on the bank of the R. Kanger, flowing through the Kanger Valley National Park (18 $\left.52^{\prime} 09^{\prime \prime} \mathrm{N} \& 8^{\circ} 56^{\prime} 05^{\prime \prime} \mathrm{E} ; 560 \mathrm{~m}\right)$ near Jagdalpur Town, Chhattisgarh State.

Other localities: None.

Sampling methods: Coring and filtration method and Direct filtration method

References: Ranga Reddy 2006: 23-37; Camacho 2006: 25.

Remark: The first cavernicolous bathynellacean species from India.

Genus Habrobathynella Schminke, 1973

30. Habrobathynella nagarjunai Ranga Reddy, 2002

Type locality: Bore well on Acharya Nagarjuna University campus $\left(16^{\circ} 22^{\prime} 41^{\prime \prime} \mathrm{N} \& 80^{\circ} 31^{\prime} 39.4^{\prime \prime} \mathrm{E} ; 19.8 \mathrm{~m}\right.$; water temperature $\left.26^{\circ} \mathrm{C} ; \mathrm{pH} 8.0\right), 13 \mathrm{~km}$ from Guntur Town, in the riparian zone of Nambur canal of R. Krishna, AP.

Sampling method: Direct filtration method

References: Ranga Reddy 2002: 38-43; Camacho 2006: 28.

Remark: Mostly confined to riparian, phreatic bore wells. 


\section{Habrobathynella schminkei Ranga Reddy, 2004}

Type locality: R. Pennar at Chennur $\left(14^{0} 34^{\prime} 00^{\prime \prime} \mathrm{N}\right.$ \& $\left.78^{\circ} 48^{\prime} 00^{\prime \prime} \mathrm{E} ; 115 \mathrm{~m}\right)$, c. $15 \mathrm{~km}$ from Kadapa Town, AP.

Other localities: River Godavari at Rajahmundry Town $\left(16^{\circ} 59^{\prime} \mathrm{N} \& 81^{\circ} 47^{\prime} \mathrm{E} ; 14 \mathrm{~m}\right)$; some bore wells in the riparian zone of R. Godavari and R. Krishna, AP.

References: Ranga Reddy 2004: 277-284 Camacho 2006: 28; Ranga Reddy \& Totakura 2010: 1-49.

\section{Habrobathynella indica Ranga Reddy \&} Schminke, 2005

Type locality: River Krishna at Vijayawada city $\left(16^{0} 31^{\prime} 8.50^{\prime \prime} \mathrm{N}\right.$ \& $\left.80^{\circ} 37^{\prime} 17.38^{\prime \prime} \mathrm{E} ; 11.8 \mathrm{~m}\right)$, close to southern end of Kanaka Durga Varadhi, a road-bridge.

Other localities: None.

Sampling method: Coring and filtration method.

References: Ranga Reddy \& Schminke 2005a: 22172224; Camacho 2006: 28; Ranga Reddy \& Totakura 2010: 40, 46-47.

Remark: This is a rare, typically hyporheic taxon, but has gone extinct at the type locality due to the increasing discharge of domestic effluents.

\section{Habrobathynella plenituda Ranga Reddy \&} Schminke, 2009

Type locality: River Godavari at Rajahmundry town $\left(16^{0} 59^{\prime} \mathrm{N} \& 8^{\circ} 47^{\prime} \mathrm{E} ; 14 \mathrm{~m}\right), \mathrm{EDG}, \mathrm{AP}$.

Other localities: None.

Sampling method: Coring and filtration method.

Reference: Ranga Reddy \& Schminke 2009b: 477485

34. Habrobathynella krishna Ranga Reddy \& Totakura, 2010

Type locality: R. Krishna at Ramannapeta Village $\left(16^{\circ} 45^{\prime} 32^{\prime \prime} \mathrm{N}\right.$ \& $80^{\circ} 07^{\prime} 35^{\prime \prime} \mathrm{E}$; $39 \mathrm{~m}$; water temperature $\left.28^{\circ} \mathrm{C} ; \mathrm{pH} 7.5\right)$, Guntur District, AP.

Other locality: R. Krishna at Madipadu Village $\left(16^{\circ} 48^{\prime} 50^{\prime \prime} \mathrm{N} \& 80^{\circ} 04^{\prime} 22^{\prime \prime} \mathrm{E}, 40 \mathrm{~m}\right)$, Guntur District, AP.

Sampling method: Coring and filtration method.

Reference: Ranga Reddy \& Totakura 2010: 4-12.

\section{Habrobathynella vaitarini Ranga Reddy \&} Totakura, 2010

Type locality: R. Krishna at Madipadu Village $\left(16^{\circ} 48^{\prime} 50^{\prime \prime} \mathrm{N}\right.$ \& $80^{\circ} 04^{\prime} 22^{\prime \prime} \mathrm{E}$; $40 \mathrm{~m}$; water temperature $32^{\circ} \mathrm{C}$; pH 7.5), Guntur District, AP.

Other localities: R. Krishna at Pulichintala $\left(16^{\circ} 49^{\prime} 22^{\prime \prime} \mathrm{N}\right.$ \& $80^{\circ} 04^{\prime} 03^{\prime \prime} \mathrm{E}$; $44 \mathrm{~m}$; water temperature $32^{\circ} \mathrm{C}$; $\mathrm{pH} 7.5$ ) and Challagariga Village $\left(16^{\circ} 45^{\prime} 32^{\prime \prime} \mathrm{N} \& 80^{\circ} 07^{\prime} 35^{\prime \prime} \mathrm{E}\right.$; 39m), Guntur District, AP.
Sampling method: Coring and filtration method.

Reference: Ranga Reddy \& Totakura 2010: 13-20.

\section{Habrobathynella savitri Ranga Reddy \&} Totakura, 2010

Type locality: River Godavari at Sundarapalli Village $\left(16^{\circ} 47^{\prime} 20^{\prime \prime} \mathrm{N} \& 82^{\circ} 03^{\prime} 25^{\prime \prime} \mathrm{E} ; 14 \mathrm{~m}\right.$; water $30^{\circ} \mathrm{C}$; $\left.\mathrm{pH} 7.5\right)$, EGD, AP.

Other localities: R. Godavari at Dhawaleswaram Town $\left(16^{\circ} 48^{\prime} 09^{\prime \prime} \mathrm{N} \& 80^{\circ} 04^{\prime} 18^{\prime \prime} \mathrm{E} ; 27 \mathrm{~m}\right)$, and Kapileswarapuram Village $\left(16^{\circ} 41^{\prime} 26^{\prime \prime} \mathrm{N} \& 82^{\circ} 02^{\prime} 24^{\prime \prime} \mathrm{E} ; 23 \mathrm{~m}\right)$, EGD, AP.

Sampling method: Coring and filtration method.

Reference: Ranga Reddy \& Totakura 2010: 20-28.

\section{Habrobathynella vidua Ranga Reddy \& Totakura,} 2010

Type locality: Farm bore at Tadepalli Village $\left(16^{\circ} 41^{\prime} 32^{\prime \prime} \mathrm{N}\right.$ \& $82^{\circ} 02^{\prime} 24^{\prime \prime} \mathrm{E} ; 21 \mathrm{~m}$; water temperature $26^{\circ} \mathrm{C}$; pH 8.0) near Vijayawada City, A.P.

Other locality: Farm bore at Kunchanapalli Village $\left(16^{\circ} 23^{\prime} 42^{\prime \prime} \mathrm{N} \& 80^{\circ} 32^{\prime} 28^{\prime \prime} \mathrm{E} ; 26 \mathrm{~m}\right.$; water temperature $\left.27^{\circ} \mathrm{C} ; \mathrm{pH} 7.5\right), 3 \mathrm{~km}$ from the type locality.

Sampling method: Direct filtration method.

Reference: Ranga Reddy \& Totakura 2010: 29-34.

Remark: So far known only from the farm bores of the riparian zone of River Krishna, AP.

38. Habrobathynella borraensis Ranga Reddy, Shabuddin \& Totakura, 2014

Type locality: The Borra Caves $\left(18^{0} 16^{\prime} 49^{\prime \prime} \mathrm{N}\right.$ \& $83^{\circ} 2^{\prime} 19^{\prime \prime} \mathrm{E}$; c. $705 \mathrm{~m}$ ) located on the East Coast of India, in the Ananthagiri Hills of the Araku Valley in the Visakhapatnam District of AP.

Other locality: None.

Sampling method: Coring and filtration method.

Reference: Ranga Reddy, Shabuddin \& Totakura 2014: (online access).

Remark: A second cavernicolous bathynellacean from India.

Genus Parvulobathynella Schminke, 1973

39. Parvulobathynella distincta Ranga Reddy, Elia \& Totakura, 2011

Type locality. R. Godavari at Kapileswarapuram $\left(16^{\circ} 47^{\prime} 28.5^{\prime \prime} \mathrm{N}\right.$ \& $82^{\circ} 03^{\prime} 33.8^{\prime \prime} \mathrm{E}$; $34.3 \mathrm{~m}$; temperature $\left.24^{\circ} \mathrm{C} ; \mathrm{pH} 7.0\right)$, c. $35 \mathrm{~km}$ from Rajahmundry town, EGD, AP.

Other localities: R. Godavari at Dhawaleswarapuram $\left(16^{\circ} 56^{\prime} 78.3^{\prime \prime} \mathrm{N}\right.$ \& $81^{\circ} 46^{\prime} 70.2^{\prime \prime} \mathrm{E} ; 40.3 \mathrm{~m}$; temperature $\left.27^{\circ} \mathrm{C} ; \mathrm{pH} 7.5\right)$ and Atreyapuram $\left(16^{\circ} 50^{\prime} 2.97^{\prime \prime} \mathrm{N}\right.$ \& $\left.81^{\circ} 47^{\prime} 12.85^{\prime \prime} \mathrm{E}\right), \mathrm{EGD}, \mathrm{A}$ P.

Sampling methods: Karaman \& Chappuis method, 
and Coring and filtration method.

Reference: Ranga Reddy, Elia \& Totakura 2011: 486494.

40. Parvulobathynella projectura Ranga Reddy, Elia \& Totakura, 2011

Type locality: River Godavari at Kotipalli Village $\left(16^{\circ} 41^{\prime} 33.5^{\prime \prime} \mathrm{N} \& 82^{\circ} 03^{\prime} 45.5^{\prime \prime} \mathrm{E} ; 10.8 \mathrm{~m}\right.$; temperature $\left.28^{\circ} \mathrm{C} ; \mathrm{pH} 7.5\right), \mathrm{EGD}, \mathrm{AP}$.

Other localities: None.

Sampling methods: Karaman \& Chappuis method and Coring and filtration method.

Reference: Ranga Reddy, Elia \& Totakura 2011: 494500.

41. Parvulobathynella macrodentata Ranga Reddy \& Totakura, 2012

Type locality: Farm bore at Peravaram village $\left(16^{\circ} 53^{\prime} 42.0^{\prime \prime} \mathrm{N} \&\right.$ \& 81045'09.8” $\mathrm{E} ; 17.7 \mathrm{~m}$; water temperature $26^{\circ} \mathrm{C} ; \mathrm{pH} 7.0$ ), ca. $20 \mathrm{~km}$ from Rajahmundry, in the riparian zone of River Godavari.

Other localities: Farm bore at Ravulapalem Village $\left(16^{\circ} 06^{\prime} 33.4^{\prime \prime} \mathrm{N} \& 81^{\circ} 46^{\prime} 49.9^{\prime \prime} \mathrm{E} ; 37 \mathrm{~m}\right.$; depth c. $10 \mathrm{~m}$; water temperature $27^{\circ} \mathrm{C}$; $\mathrm{pH} 7.0$ ), $20 \mathrm{~km}$ from Rajahmundry, East Godavari District and also at Mamillapalli Village $\left(16^{\circ} 02^{\prime} 23.8^{\prime \prime} \mathrm{N}\right.$ \& 8032'35.4”E; 32m) near Nidubrolu town, Guntur District, AP.

Sampling method: Direct filtration.

Reference: Ranga Reddy \& Totakura 2012b: 871-882.

Remark: Mostly confined to the aquifers of the riparian areas.

\section{DISCUSSION}

In India, both the Himalayan and Peninsular River Systems present vast and ecologically diversified hyporheic realm and riparian areas, apparently harboring enormous biodiversity. However, as already mentioned in the Introduction, little is known about the Indian hyporheic biota. This is due to the minute size of most of the organisms, the difficulties involved in their sampling, the exacting microscopic study and drawing work, the lack of taxonomic expertise and funding support, etc.

The present faunistic survey covering only a fraction of the Indian hyporheic and riparian realm is indeed rewarding in that it has yielded 41 copepod and bathynellacean species, of which as many as 31 species are new to science and formally described and the remaining one are previously known in the literature; an additional 20 new species in the samples are yet to be named and described. The eumalacostracan order Bathynellacea and the harpacticoid copepod family Parastenocarididae are two significant, major groups of stygofauna that have been recorded for the first time from India. Both these groups are of much value in historical biogeography and phylogenetic studies. In particular, the Bathynellacea represents one of the oldest freshwater crustacean groups whose ancestors inhabited the seas in the Carboniferous or even earlier, now absent from the epigean realm. This group as a whole might have achieved its worldwide distribution prior to the breakup of Pangaea, and its present biogeography can be more convincingly explained by the vicariance model rather than by the classical dispersal model (Schminke 1974; Schram 1977, 2008). It is noteworthy that while all the Indian bathynellacean taxa are distinctly different from their Asian counterparts, they display spectacular Gondwanan heritage (Ranga Reddy 2011b). Of the two Indian endemic genera Serbanibathynella and Indobathynella, the latter is the most derived one in the family Bathynellidae. The parabathynellid genus Habrobathynella is remarkably speciose with as many as 12 Indian species (three new species present in the samples are yet to be named and described), nine of them inhabiting the sandy sediments of peninsular rivers. This genus is known outside India only by two species in Madagascar.

According to Noodt (1969), compared with the Bathynellacea, the Parastenocarididae is a much younger group, having originated possibly in the early Tertiary or even earlier. However, because parastenocaridids have no marine relatives or modern pathways between different continents (Boxshall \& Jaume 2000), it has been postulated that they have a Pangaean origin (Karanovic 2006). The latter taxon is as yet known by 11 species in India. While eight species are distributed in alluvial sediments, one species each is restricted to a cave and two to riparian borewells. Two genera, viz., Kinnecaris and Siolicaris, have distinct Gondwanan affinities and so do three cyclopoid copepod genera, viz., Haplocyclops, Rybocyclops and Allocyclopina as well (Ranga Reddy 2011b). Since the Gondwanan lineages represent the remnants of unique ancient biota (Mani 1974; Roelants et al. 2004), they require urgent attention from conservationists in order that the overall evolutionary history of Indian biota is preserved (Karanth 2006). All in all, these tiny ancient crustaceans inhabiting the sandy sediments are no less important than the spectacular epigean vertebrates in understanding the evolutionary history of the earth's crust.

It is also worthy of note that amongst the other 
harpacticoid copepod species, Delavalia madrasensis, Folioquinpes chathamensis, Neomiscegenus indicus, and Mesochra wolskii belong to the almost exclusively marine families. Clearly, the occurrence of these species in the truly freshwater conditions of the hyporheic zone of the river Krishna near Vijayawada is indicative of their remarkable euryhaline adaptations.

\section{CONSERVATION}

Construction boom in the wake of rapid urbanization has fuelled increasing demand for river sand. As a result, all the Indian rivers without exception have been and are still literally plundered of their alluvium on a large scale. Sand miners are digging to a depth of about $15 \mathrm{~m}$ with the help of machines, and even extracting the earth after touching the river floor. Besides the staggering and visible on-site and off-site ill-effects of uncontrolled sand extraction such as channel degradation and erosion, deepening of rivers and enlargement of river mouths, lowering of water tables in the nearby riparian areas plus occasional saline-water intrusion from the nearby seas, infrastructure damage like undermining of bridges and other structures, etc. (see Kondolf \& Swanson 1993; Kondolf 1997; Mori et al. 2011), and sadly and more importantly, the highly fragile hyporheic habitats and their associated biota are gouged out along with their homes, as it were. In this connection, it is also noteworthy that the dubious 'eco-friendly' policy announced by certain state governments, providing for sand extraction up to $2 \mathrm{~m}$, is utterly myopic and disastrous to sand-associated life because most of the hyporheic life is confined to the upper one meter or so of the sediment. This fact must be taken cognizance of by the policy makers.

Considering the ecological importance of the hyporheic biodiversity in riverine ecosystem functioning, total ban must be imposed on sand mining activities. Should this be not feasible, at least certain tracts of each of our river banks must be given legal protection against the sand mafia so that such protected corridors could ensure the regeneration and preservation of the hyporheic biota. Simultaneously, immediate steps need be taken to encourage research activities leading to the finding of suitable, low-cost and easily available alternatives to river sand for construction industry. In view of the importance of hyporheic science as a multidisciplinary area of specialization, funding agencies in the country will do well to play a pro-active role in encouraging research in this area, starting from the taxonomic characterization of species.

\section{REFERENCES}

Benke, A.C. \& J.B. Wallace (1980). The trophic basis of production among net-spinning caddisflies in a southern Applachian stream. Ecology 61: 278-298.

Borchardt, M.A. \& T.L. Bott (1995). Meiofaunal grazing of bacteria and algae in a Piedmont stream. Journal of the North American Benthological Society 14: 278-298.

Boulton, A.J. (2000a). Subsurface macrofauna, pp. 337-361. In: Jones, J.B. \& P.J. Mulholland (eds.). Streams and Ground Waters, Academic Press, London, 417pp.

Boulton, A.J. (2000b). River ecosystem health down under: assessing ecological condition in riverine groundwater zones in Australia. Ecosystem Health 6: 108-118.

Boulton, A.J., T. Datry, T. Kasahara, M.Mutz \& J.A. Stanford (2010). Ecology and management of the hyporheic zone: streamgroundwater interactions of running waters and their floodplains. Journal of the North American Benthological Society 29: 26-40; http://dx.doi.org/10.1899/08-017.1

Boxshall, G.A. \& D. Jaume (2000). Making waves: the repeated colonization of freshwater by copepod crustaceans. Advances in Ecological Research 31: 61-79.

Brown, A.V., R.L. Limbeck \& M.D. Schram (1989). Trophic importance of zooplankton in streams with alluvial riffle and pool geomorphometry. Archiv fúr Hydrobiologie 114: 349-367.

Bruno, M.C. \& V. Cottarelli (1999). Harpacticoids from groundwaters in the Philippines: Parastenocaris mangyans, new species, Epactophanes philippinus, new species and redescription Phyllognathopus bassoti (Copepoda). Journal of Crustacean Biology 19(3): 510-529.

Camacho, A.I. (2006). An annotated checklist of the Syncarida (Crustacea, Malacostraca) of the world. Zootaxa 1374: 1-54.

Chappuis, P.A. (1942). Eine Methode zur Untersuchung der Grundwasserfauna. Acta Scientiarum Mathematicarum Naturalium (Unviersitatis Francisco-Josephina) Kolozsvar 6: 3-7.

Chappuis, P.A. (1954). Copépodes Harpacticoïdes des Indes et del'Iran. Bulletin de la Société d'Histoire Naturelle de Toulouse 89(3/4): 213224.

Corgosinho, P.H.C., Y. Ranga Reddy \& P.M. Arbizu (2012). Revision of the genus Siolicaris Jakobi, 1972, with redescriptions of S. sioli (Noodt, 1963) and S. jakobi (Noodt, 1963) from South America, and S. sandhya (Ranga Reddy, 2001) comb. nov. from India (Copepoda, Harpacticoida, Parastenocarididae). Zootaxa 3493: 49-71.

Cottarelli, V., M.C. Bruno \& R. Berara (2010). First record of Parastenocarididae from Thailand and description of a new genus (Copepoda, Harpacticoida). Journal of Crustacean Biology 30(1): 478-494.

Danielopol, D.L., M. Greuzé des Châtelliers, F. Moeszlacher, P. Popsi \& R. Popa (1994). Adaptation of Crustacea to interstitial habitats: a practical agenda for ecological studies, pp. 217-243. In: Gilbert, J., D.L. Danielopol \& J.A. Stanford (eds.). Groundwater Ecology, Academic Press, Inc., 571pp.

Defaye, D. \& Y. Ranga Reddy (2008). A new Allocyclopina species (Copepoda, Cyclopoida, Cyclopinidae) from a hyporheic zone of the River Godavari, India and comments on the morphological characters of the genus. Crustaceana 81(9): 1119-1141.

Dussart, B.H. \& D. Defaye (1990). Répertoire mondial des Crustacés Copépodes des eaux intérieures.III. Harpacticoides. Crustaceana (Suppl.) 16: 1-384.

Elser, J.J., D.R. Dobberfuhl, N.A. MacKay \& J.H. Schampel (1996). Organism size, life history, and N: P stoichiometry. BioScience 46: 674-684.

Enckell, P.H. (1970). Parastenocarididae (Copepoda, Harpacticoida) from Ceylon. Arkiv för Zoologi 22: 545-556. 
Environment Agency (2009). The Hyporheic Handbook, Science Report: SC050070.

Feris, K., P. Ramsey, C. Frazar, J.N. Moore, J.E. Gannon \& W.E. Holbert (2003). Differences in hyporheic-zone microbial community structure along a heavy-metal contaminant gradient. Applied and Environmental Microbiology 69: 5563-5573.

Fiers, F. \& P. Rutledge (1990). Harpacticoid copepods associated with Spartina alterniflora culms from the marshes of Cocodrie, Louisiana (Crustacea, Copepoda). Bulletin de I'Institut Royal des Sciences Naturelles de Belgique, Biologie 60: 105-125, figs. 1-9, tabs.1-3.

Galassi, D.M.P., P. De Laurentiis \& B. Fiasca (2011). Systematics of the Phyllognathopodidae (Copepoda, Harpacticoida): re-examination of Phyllognathopus viguieri (Maupas, 1892) and Parbatocamptus jochenmartensi Dumont and Maas, 1988, proposal of a new genus for Phyllognathopus bassoti Rouch, 1972, and description of a new species of Phyllognathopus. ZooKeys 104: 1-65; http://dx.doi. org/10.3897/zookeys.104.763

Gibert, J., M.J. Dole-Olivier, P. Marmonier \& P.Vervier (1990). Surface water-groundwater ecotones, pp. 199-226, In: Naiman, R.J. \& H. Décamps (eds.). The Ecology and Management of Aquatic-Terrestrial Ecotones, UNESCO, Paris and Parthenon Publishers, Carnforth, UK.

Gómez, S., J.W. Fleege, A. Rocha-Olivares \& D. Foltz (2004). Four new species of Cletocamptus Schmankewitsch, 1875, closely related to Cletocamptus deitersi (Richard, 1897) (Copepoda: Harpacticoida). Journal of Natural History 37: 2669-2732.

Hakenkamp, C.C. \& P.A. Palmer (1999). The ecology of hyporheic meiofauna, pp. 307-336. In: Jones J.B. \& P.J. Mulholland (eds.). Streams and Ground Waters, Academic Press, New York, 417pp.

Hakenkamp, C.C., A. Morin \& D.L Strayer (2002). The functional importance of meiofauna, pp. 321-335. In: Rundle, S.D., A.L. Robertson \& J.M. Schmid-Araya (eds.). Freshwater Meiofauna: Biology and Ecology. Backhuys Publishers, Leiden, 369pp.

Hessen, D.O. (1997). Stoichiometry in food webs-Lotka revisited. Oikos 79: 195-199.

Hamond, R. (1973). The harpacticoid copepods (Crustacea) of the Saline Lakes in Southeast Australia, with special reference to the Laophontidae. Records of the Australian Museum 28(17): 393-420.

ICZN (1999). International Code of Zoological Nomenclature-4th Edition. International Trust for Zoological Nomenclature, London, xxix+306pp.

Karanovic, T. (2005). Two new subterranean Parastenocarididae (Crustacea, Copepoda, Harpacticoida) from Western Australia. Records of the Western Australian Museum 22: 353-374.

Karanovic, T. (2006). Subterranean Copepods (Crustacea, Copepoda) from the Pilbara Region in Western Australia. Records of the Western Australian Museum 70(suppl.): 1-239.

Karanovic, T. \& G.L. Pesce (2001). A new genus and species of the family Ectinosomatidae (Crustacea: Copepoda: Harpacticoida) from the groundwaters of India. Annales de Limnologie 37(4): 281-292.

Karanovic, T. \& Y. Ranga Reddy (2004a). First record of Phyllognathopus bassoti Rouch, 1972 from India, with remarks on the family Phylloghathopodidae Gurney, 1932 (Crustacea, Copepoda, Harpacticoida). Annales de Limnologie 40(2): 121-132.

Karanovic, T. \& Y. Ranga Reddy (2004b). A new genus and species of the family Diosaccidae (Copepoda: Harpacticoida) from the groundwaters of India. Journal of Crustacean Biology 24(2): 246260.

Karanovic, T. \& Y. Ranga Reddy (2005). First Haplocyclops Kiefer (Crustacea, Copepoda) from Indian subterranean waters: the most reduced free-living cyclopoid. Annales de Limnologie 41(2): 83-92.

Karanth, K.P. (2006). Out-of-India Gondwanan origin of some tropical Asian biota. Current Science 90(6): 789-792.

Karaytug, S. \& G.A. Boxshall (1998). Partial revision of Paracyclops Claus, 1893 (Copepoda, Cyclopoida, Cyclopidae) with description of four new species. Bulletin of the Natural History Museum, London. (Zoology) 64(2): 111-205.

Karaytug, S. (1999). Copepoda: Cyclopoida. Genera Parayclops, Ochridiacyclops and key to the Eucyclopinae. In: Guides to the Identification of the Microinvertebrates of the Continental Waters of the World. Dumont, H. J. (coordinating ed.), 14. Backhuys Publishers, Leiden, 217pp.

Kondolf, G.M. (1997). Hungry Water: effects of dam and gravel mining on river channels. Environmental Management 21: 533-551.

Kondolf, G.M. \& M.L. Swanson (1993). Channel adjustments to reservoir construction and gravel extraction along stony creek, California. Environmental Geology and Water Science 21: 256-269.

Larned, S.T. (2012). Phreatic groundwater ecosystems: research frontiers for freshwater ecology. Freshwater Biology 57: 885-906; http://dx.doi.org/10.1111/j.1365-2427.2012.02769.x

Lang, K. (1948). Monographie der Harpacticiden. Lund: Hakan Ohlsson. Vol. I, 1-896, Vol. II, 897-1682.

Mani, M.S. (1974). Biogeographical evolution in India, pp. 698-724. In: Mani, M.S (ed.). Ecology and Biogeography in India. Dr. W. Junk b. v. Publ., The Hague, 772pp.

McNicol, R.E., E. Scherer \& E.J. Murkin (1985). Quantitative field investigations of feeding and territorial behavior of young-of-theyear brook charr, Salvelinus fontinalis. Environmental Biology of Fishes 12: 219-229.

Mori, N., T. Simčič \& S. Lukančič (2011). The effect of in-stream gravel extraction in a pre-alpine gravel-bed river on hyporheic invertebrate community. Hydrobiologia 667: 15-30; http://dx.doi. org/ DOI:10.1007/s10750-011-0648-x

Mulholland, P.J. \& R. Webster (2010). Nutrient dynamics in streams and the role of J-NABS. Journal of the North American Benthological Society 29: 100-117.

Noodt, W. (1969). Die Grundwasserfauna Sűdamerikas, Vol. 2, pp. 659-684. In: Fittkau, E., J. Illies, H. Klinge, G. H. Schwabe \& H. Sioli, (eds.). Biogeography and Ecology in South America. Dr. W. Junk N. V., The Hague.

Notenboom, J., S. Plénet \& M.J. Turquin (1994). Groundwater contamination and its impact on groundwater animals and ecosystems, pp. 477-504. In: Gibert, J., D.L. Danielopol \& J.A. Stanford (eds.). Groundwater Ecology, Academic Press, London, $571 \mathrm{pp}$.

Odum, E.P. (1971). Fundamentals of Ecology, W B. Saunders Co., Philadelphia, 574pp.

Orghidan, T. (1959). Ein neuer Lebensraum des unterirdischen Wassers: der hyporhischen Biotop. Archiv für Hydrobiologie 55: 392-414

Palmer, M.A. (1990). Temporal and spatial dynamics of meiofauna within the hyporheic zone of Goose Creek, Virginia Journal of the North American Benthological Society 9: 17-25.

Pennak, R.W. (1940). Ecology of the microscopic Metazoa inhabiting the sandy beaches of some Wisconsin lakes. Ecoogical Monographs 10: 537-615.

Radhakrishna, Y. \& Y. Ranga Reddy (1978). A new species of Stenhelia Boeck (Copepoda, Harpacticoida) from South India. Crustaceana 35(2): 152-158.

Ranga Reddy, Y. (2001). Discovery of Parastenocarididae (Copepoda, Harpacticoida) in India, with the description of three new species of Parastenocaris Kessler, 1913, from the River Krishna at Vijayawada. Crustaceana 74(8): 705-733.

Ranga Reddy, Y. (2002). Habrobathynella nagarjunai $\mathrm{n}$. sp., the second representative of Bathynellacea (Crustacea, Syncarida) from groundwaters of South India. Hydrobiologia 470: 37-43.

Ranga Reddy, Y. (2004). Existence of the Order Bathynellacea (Crustacea, Syncarida) in South Asia: a new species of the genus Habrobathynella Schminke from River Pennar, South India. Journal of the Bombay Natural History Society 101: 277-284.

Ranga Reddy, Y. (2006). First Asian report of the genus Chilibathynella Noodt, 1963 (Bathynellacea, Syncarida), with the description and biogeographic significance of a new species from Kotumsar Cave, India. Zootaxa 1370: 23-37.

Ranga Reddy, Y. (2011a). Two new hyporheic Parastenocarididae from India: Parastenocaris sutlej n. sp. and P. gundlakamma n. sp. (Copepoda, Harpacticoida). Crustaceana Monographs 16: 461-487.

Ranga Reddy, Y. (2011b). Gondwanan heritage in groundwater crustaceans of peninsular India. Current Science 101: 156-158. 
Ranga Reddy, Y. (2011c). A new phreatic species of genus Parastenocaris Kessler (Copepoda: Harpacticoida: Parastenocarididae) from southeastern India, with a key to species of Indian subcontinent. Biosystematica 5: 21-29.

Ranga Reddy, Y. \& D. Defaye (2007). Parastenocarididae (Crustacea, Copepoda, Harpacticoida of India: description of Parastenocaris mahanadi n. sp., and redescription of P. curvispinus Enckell, 1970 from hyporheic habitats. Zootaxa 1580: 1-26. (Erratum: Zootaxa 1593: 68)

Ranga Reddy, Y. \& D. Defaye (2008). Discovery of the genus Rybocyclops Dussart, 1982 (Crustacea, Copepoda, Cyclopoida) in subterranean groundwaters of southeastern India, with description of a new species and its biogeographic significance. Zootaxa 1810: $40-50$.

Ranga Reddy, Y. \& D. Defaye (2009). Two new Parastenocarididae (Copepoda, Harpacticoida) from India: Parastenocaris muvattupuzha $\mathrm{n}$. $\mathrm{sp}$. from a river and $P$. kotumsarensis $\mathrm{n}$. $\mathrm{sp}$. from a cave. Zootaxa 2077: 31-55

Ranga Reddy, Y. \& Y. Radhakrishna (1979). A new record of Cletocampus deitersi (Richard, 1895) (Copepoda: Harpacticoida) from India. Current Science 48(1): 45

Ranga Reddy, Y. \& H.K. Schminke (2005a). Morphological diversity of habrobathynellids (Parabathynellidae, Bathynellacea) in India, with the description of a new species. Journal of Natural History 39(24): 2217-2224.

Ranga Reddy, Y. \& H.K. Schminke (2005b). A new bathynellid from India with unusual mouthparts (Bathynellacea: Bathynellidae). Journal of Crustacean Biology 25(1): 25-30.

Ranga Reddy, Y. \& H.K. Schminke (2009a). Discovery of the genus Kinnecaris Jakobi, 1972 (Copepoda, Harpacticoida, Parastenocarididae) in southeastern India, with description of a new species. Crustaceana 82(3): 311-326.

Ranga Reddy, Y. \& H.K. Schminke (2009b). Co-occurrence of two species of the genus Habrobathynella Schminke, 1973 (Malacostraca, Bathynellacea) in sandy sediments of the River Godavari, southeastern India, with the description of a new species. Crustaceana 82(4): 475-485.

Ranga Reddy, Y. \& V.R. Totakura (2010). A taxonomic revision of the genus Habrobathynella Schminke, 1973, with the description of four new species from southeastern India (Crustacea, Malacostraca, Bathynellacea). Zootaxa 2532: 1-54.

Ranga Reddy, Y. \& V.R. Totakura (2012a). Indobathynella prehensilis gen. et sp. nov., an aberrant species of Bathynellacea (Eumalacostraca) from India. Journal of Crustacean Biology 32(2): 281-293; http://dx.doi.org/10.1163/193724011X615587

Ranga Reddy, Y. \& V.R. Totakura (2012b). A new phreatic species of the genus Parvulobathynella (Malacostraca: Bathynellacea) from southeastern India, along with an updated key to the species. Journal of Crustacean Biology 32(5): 871-882.

Ranga Reddy, Y., J. Drewes \& H.K. Schminke (2008). A new species of the genus Atopobathynella Schminke, 1973 (Crustacea, Syncarida, Bathynellacea) from the hyporheic zone of the River Godavari, South India. Zootaxa 1829: 52-60.
Ranga Reddy, Y., B. Elia \& V.R. Totakura (2011). First Asian record of the genus Parvulobathynella (Malacostraca, Bathynellacea) with description of two new species from southeastern India and amendment of the generic diagnosis. Journal of Crustacean Biology 31(3): 485-508; http://dx.doi.org/10.1651/10-3435.1

Ranga Reddy, Y., S. Shabuddin \& V.R. Totakura (2014). Habrobathynella borraensis n. sp. (Syncarida, Bathynellacea; Parabathynellidae) from the Borra Caves of southeastern India, with a note on the taxonomic significance of paragnath morphology. Journal of Crustacean Biology 34(1): 90-106; http://dx.doi.org/10.1163/1937240X-00002210

Roelants, K., J. Jiang \& S. Bassuyt (2004). Endemic ranid (Amphibia: Anura) genera in southern mountain ranges of the Indian subcontinent represent ancient frog lineages: evolution from molecular data. Molecular Phylogenetics and Evolution 31: 730-740.

Rouch, R. (1972). Deux harpacticidae nouveaux de l'ile de Long Island (Territoire de Papouasie et de Nouvelle-Guinée). Archives Zoologie Expérimentale \& Générale 113(1): 147-164.

Schmid-Araya, J.M. \& P.E. Schmid (2000). Trophic relationships: integrating meiofauna into a realistic benthic food web. Freshwater Biology 44: 149-164.

Schminke, H.K. (1974). Mesozoic intercontinental relationships as evidenced by bathynellid Crustacea (Syncarida: Malacostraca). Systematic Zoology 23: 157-164.

Schminke, H.K. (2010). High level phylogenetic relationships within Parastenocarididae (Copepoda, Harpacticoida). Crustaceana 83(3): 343-367.

Schmith, L.C. \& L.A. Smock (1992). Ecology of invertebrate predators in a coastal plain streams. Freshwater Biology 28: 319-329.

Schram, F.R. (1977). Paleozoogeography of late Paleozoic and Triassic Malacostraca. Systematic Zoology 26: 367-379.

Schram, F.R. (2008). Does biogeography have a future in a globalized world with globalized faunas? Contributions to Zoology 77: 127-133.

Sewell, R.B.S. (1924). Fauna of the Chilka Lake. Crustacea Copepoda. Memoirs of the Indian Museum, Calcutta 5: 771-551.

Stanford, J.A. \& J.V. Ward (1993). An ecosystem perspective of alluvial rivers: connectivity and the hyporheic corridor. Journal of the North American Benthological Society 12: 48-60.

Vervier, P., J. Gibert, P. Marmonier \& M.J. Dole-Olivier (1992). A perspective on the permeability of the surface freshwatergroundwater ecotone. Journal of the North American Benthological Society 11: 93-102.

Wells, J.B.J. (1971). The Harpacticoida (Crustacea: Copepoda) of two beaches in south-east India. Journal Natural History 5: 507-520.

Wells, J.B.J. (2011). An annotated checklist and keys to the species of Copepoda Harpacticoida (Crustacea). Zootaxa 1568: 1-872.

Wells, J.B.J. \& G.C. Rao (1987). Littoral Harpacticoida (Crustacea: Copepoda) from Andaman and Nicobar Islands. Memoirs of the Zoological Survey of India 16(4): 1-385.

Williams, D.D. (1981). The first diets of the post-emergent brook trout (Salvelinus fontinalis) and Atlantic salmon (Salmo salar alevins) in a Quebec river. Canadian Journal of Fisheries and Aquatic Sciences 38: 765-771.

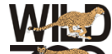

